\title{
Pectus excavatum and congenital cystic lung lesion: simultaneous surgery advocated
}

\author{
Hui Wang ${ }^{1,2 \#}$, Feng-Hua Wang ${ }^{1 \#}$, Jian-Hua Liang ${ }^{1 \#}$, Wei Liu ${ }^{1}$, Jing-Zhou Yang ${ }^{3}$, Xiao-Hua Guo ${ }^{1,2}$, Wen- \\ Yue $\mathrm{Si}^{3}$, Hui-Min Xia ${ }^{1,2}$ \\ ${ }^{1}$ Department of Pediatric Surgery, Guangzhou Women and Children's Medical Center, Guangzhou Medical University, Guangzhou 510623, China; \\ ${ }^{2}$ Southern Medical University, Guangzhou 510515, China; ${ }^{3}$ Institute of Pediatrics, Guangzhou Women and Children's Medical Center, Guangzhou \\ Medical University, Guangzhou 510623, China \\ Contributions: (I) Conception and design: H Wang, W Liu, HM Xia; (II) Administrative support: W Liu, HM Xia; (III) Provision of study materials \\ or patients: H Wang, FH Wang, JH Liang, W Liu; (IV) Collection and assembly of data: H Wang, XH Guo; (V) Data analysis and interpretation: \\ WY Si, JZ Yang; (VI) Manuscript writing: All authors; (VII) Final approval of manuscript: All authors. \\ "These authors contributed equally to this work. \\ Correspondence to: Hui-Min Xia; Wei Liu. Department of Pediatric Surgery, Guangzhou Women and Children's Medical Center, 9 Jinsui Road, \\ Guangzhou 510623, China. Email: xia-huimin@foxmail.com; liuwei19610624@126.com.
}

Background: Severe pectus excavatum (PE) may be concomitant with congenital cystic lung lesions (CCLLs) that also require surgery. It is ideal to correct these two deformities concurrently, but the safety and efficacy of a simultaneous surgical technique remain unknown.

Methods: Between 2007 and 2017, 635 patients with severe PE were admitted at our medical center. Eight patients underwent minimally invasive repair of PE and lobectomy simultaneously. The patient characteristics and operative data were analyzed and compared with another group of patients who underwent lobectomy alone for contemporaneous CCLLs.

Results: The severity of PE (mean Haller index 5.70) and CCLLs were confirmed by computed tomography (CT). Simultaneous minimally invasive repair and lobectomy were performed successfully. There were no significant differences in the mean blood loss $(14 \mathrm{~mL} / \mathrm{kg})$, the mean weaning time from mechanical ventilation (900 minutes) and the mean hospital stay (16 days) $(\mathrm{P}>0.05)$. The mean operative time (170 minutes) was extended, as expected $(\mathrm{P}=0.02)$. With a mean follow-up of 22 months, the overall cosmetic results were good.

Conclusions: Simultaneous minimally invasive repair and lobectomy appears to be a technically safe and reliable method for the treatment of concurrent PE and CCLLs, although further studies are needed in the long-term follow-up.

Keywords: Pectus excavatum (PE); congenital cystic lung lesion (CCLL); children; minimally invasive repair; technique

Submitted Feb 20, 2018. Accepted for publication Sep 18, 2018.

doi: $10.21037 /$ jtd.2018.10.8

View this article at: http://dx.doi.org/10.21037/jtd.2018.10.8

\section{Introduction}

Pectus excavatum (PE) is the most common anterior chest wall deformity (1). PE is characterized by the depression of the anterior chest wall, and this condition has been associated with cardio-pulmonary compression symptoms and the presence of restricted pulmonary ventilation disorders. Although most patients are asymptomatic, both the residual volume and the ratio of residual volume/total lung capacity increase in all PE patients, which may be due to air-trapping and impaired lung growth $(2,3)$. In terms of the pathophysiological mechanisms mentioned above, 
Table 1 Patient characteristics

\begin{tabular}{|c|c|c|c|c|c|c|c|}
\hline Patient No. & $\begin{array}{l}\text { Age (years/ } \\
\text { months) }\end{array}$ & $\begin{array}{l}\text { Sex (male/ } \\
\text { female) }\end{array}$ & Weight (kg) & Symptoms & Haller index & $\begin{array}{l}\text { Length of stay } \\
\text { (days) }\end{array}$ & $\begin{array}{l}\text { Follow-up } \\
\text { (months) }\end{array}$ \\
\hline 1 & 4 months & Female & 5 & RRTI, dyspnea & 3.28 & 14 & 25 \\
\hline 2 & 8 months & Male & 6 & RRTI, dyspnea & 4.22 & 15 & 34 \\
\hline 3 & 3 years & Male & 10 & RRTI, dyspnea & 7.19 & 26 & 39 \\
\hline 6 & 8 years & Female & 22 & RRTI, dyspnea & 4.04 & 9 & 10 \\
\hline 7 & 8 years & Male & 18 & RRTI, incapacitated & 10.14 & 18 & 2 \\
\hline 8 & 8 months & Male & 6.5 & RRTI, dyspnea & 5.99 & 18 & 3 \\
\hline
\end{tabular}

RRTI: recurrent respiratory tract infection.

$\mathrm{PE}$ has something in common with congenital cystic lung lesions (CCLLs), which include congenital pulmonary cysts, congenital cyst adenomatoid malformations, pulmonary sequestration, and congenital lobar emphysema.

$\mathrm{PE}$ is usually an isolated congenital deformity with an incidence of 1 in 300 births (4). Severe PE may occasionally, as evidenced by the $0.49 \%$ incidence rate in eastern China, be concomitant with CCLL, and it requires surgery (5). It is ideal to correct the two deformities concurrently. However, the safety and efficiency of a simultaneous surgical technique remain unknown. Minimally invasive repair of pectus excavatum (MIRPE) using the Nuss procedure has been performed at our medical center since 2007 . To date, 635 patients have been admitted for severe PE. Eight $(1.26 \%)$ of these patients underwent simultaneous lobectomy and subsequent PE correction. In this study, we report the findings over a 10 -year period. The patient characteristics and operative data were analyzed and compared with another group of patients who underwent lobectomy alone for contemporaneous CCLL.

\section{Methods}

\section{Patients}

Severe PE was defined as having a Haller index higher than 3.25. Overall, 635 patients underwent MIRPE at our center between 2007 and 2017. In this study, patients who underwent simultaneous surgery for MIRPE and lobectomy were included, while patients who underwent MIRPE or lobectomy separately for either lesion were excluded from this retrospective study. As a result, 8 patients were included in this study and are represented as cases 1-8 based on their admission sequence (Table 1). All patients were provided informed consent and signed the consent form. Patient characteristics and symptoms were as follows: cases 1, 2 and 8 were infants, and their symptoms were polypnea and recurrent respiratory tract infection; cases 3, 4, 5, 6 and 7 were children, and their symptoms included declined body endurance, fatigue, recurrent respiratory tract infections, and slow body growth. Physical examination findings showed that the sternum, particularly the middle and lower portions, sank back into the chest in all of the patients. The objective criteria for the study included a computed tomography (CT) scan that showed PE severity, cystic lung lesions, and cardiac displacement. The mean Haller index was 5.70 (range, 3.2810.14). Additionally, the ECG results for one case showed an abnormal $\mathrm{T}$ wave, and the cardiac ultrasonography results for one case showed mitral valve prolapse. The patient characteristics and operative data were analyzed and compared with those of another 30 patients who underwent lobectomy alone for contemporaneous CCLL.

\section{Surgical technique}

Simultaneous lobectomy with subsequent MIRPE were performed (Figure 1). Briefly, the patient was first placed in lateral recumbency. An incision was made on the posterior lateral side of the chest wall and a lobectomy was performed. After the lobectomy, MIRPE was performed with the patient in a supine position. Through two lateral 

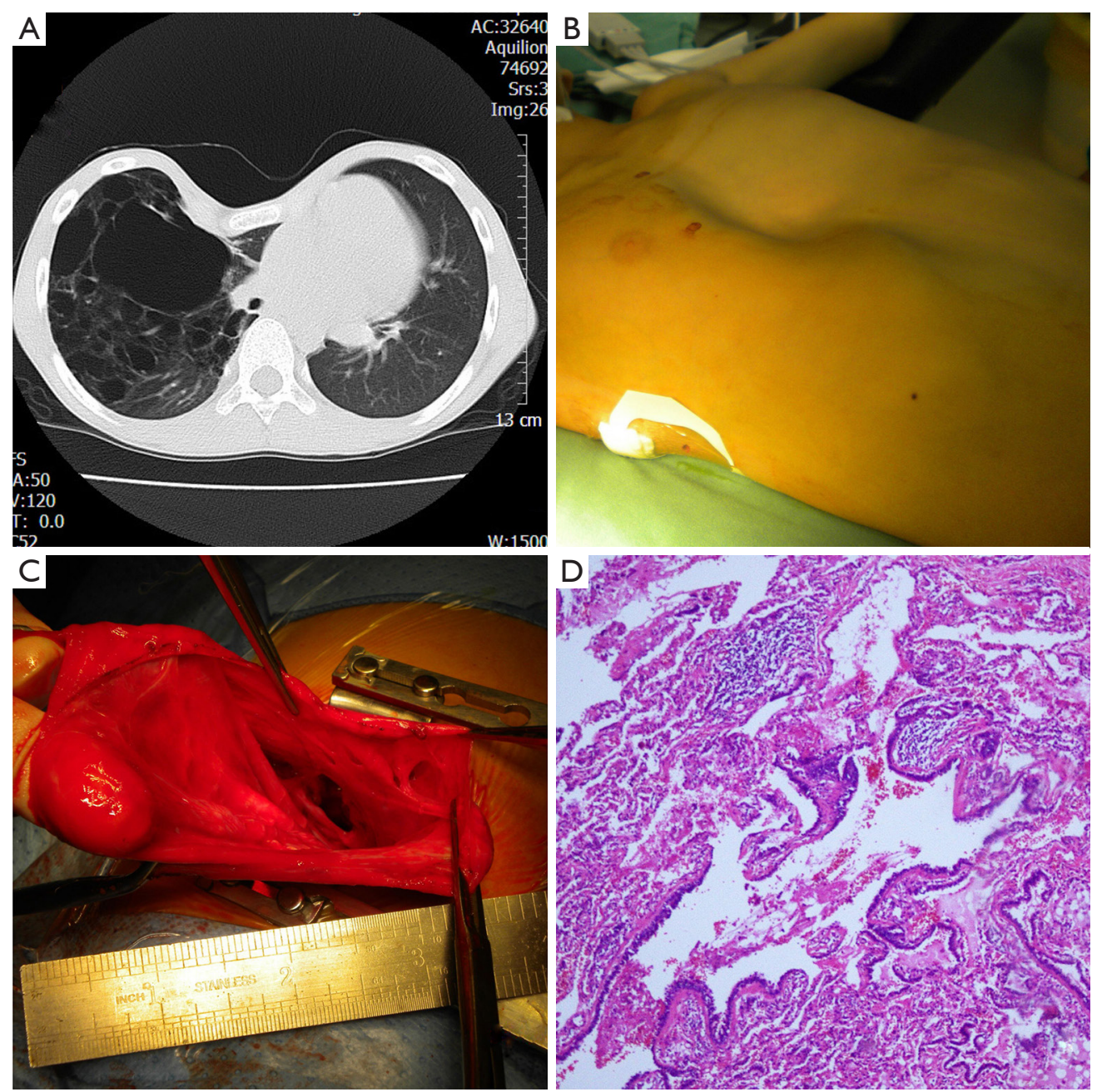

Figure 1 Images of case 5. A preoperative CT image (A) showing a right cystic lung lesion and severe PE deformity (Haller index =5.71) with obvious heart displacement due to compression. An intra-operative image (B) showing the typical signs of severe PE. Gross (C) and histological (D) examinations confirmed cystic lung lesions (H\&E, 100×).

thoracic incisions, a pre-bent bar (Grikin Inc., Beijing or Biomet, Jacksonville, FL, USA) matching the sidewalls of the chest was inserted into the posterior of the sternum with thoracoscopic guidance. Then, the Nuss bar was rotated to elevate the depressed sternum. When the cosmetic results were satisfactory, the bar was fixed to stabilizers by using stainless steel wires. A drainage pipe was placed on side of the chest wall with the lung lesion. The operative outcome was evaluated by the duration of surgery, the duration of ventilator weaning, and perioperative bleeding. Chest drainage tubes were removed 2-3 days after surgery. When post-operative thoracic radiography indicated that the steel bars were firmly fixed and lung recruitment was satisfactory without obvious symptoms, patients were released from the hospital.

\section{Video legends (Figure 2)}

The severity of pectus excavatum and congenital cystic lung lesions were confirmed by computed tomography. Simultaneous minimally invasive repair and lobectomy were performed successfully. Briefly, the patient was first placed in lateral recumbency. The incision was made on the posterior lateral side of the chest wall, and lobectomy was performed. After lobectomy, MIRPE was performed with the patient in a supine position. Through two lateral thoracic incisions, a pre-bent bar (Grikin Inc., Beijing or Biomet, Jacksonville, FL, USA) matching the sidewalls of the chest was inserted into the posterior of the sternum under thoracoscopic guidance. Then, the Nuss bar was rotated to elevate 
the depressed sternum. When the cosmetic results were satisfactory, the bar was fixed to stabilizers by using stainless steel wires. Drainage tubes were placed on the side of the chest wall with the lung lesion.

\section{Statistical analysis}

All data were expressed as the mean \pm SD. The two-tailed $t$-test was performed to compare the means, and $\mathrm{P}<0.05$ indicated statistical significance.

\section{Statement of ethics approval}

The study was approved by the institutional review board of the medical center (No. 2016111876).

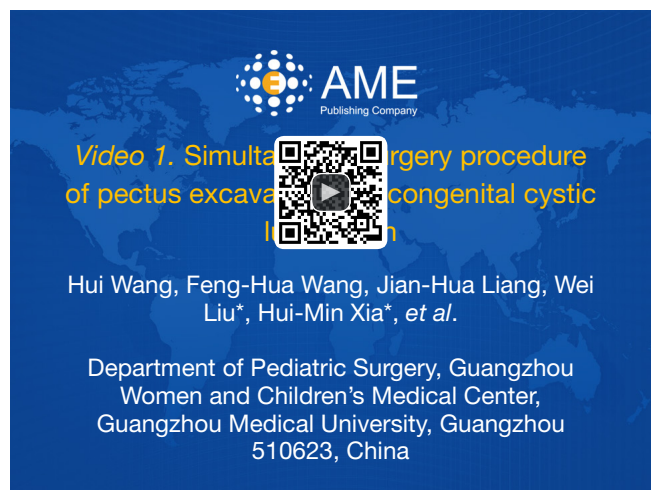

Figure 2 Simultaneous surgery procedure of pectus excavatum and congenital cystic lung lesion (6).

Available online: http://www.asvide.com/article/view/28462

\section{Results}

Cases 1, 2 and 8 were infants, and they were 4, 8, and 8 months respectively.

While cases 3, 4, 5, 6 and 7 were children, and their ages were 3 years, 1 year and 9-month, 13, 8 and 8 years. The severity of PE (Haller index range 3.28-10.14; mean 5.70) and CCLLs were confirmed by CT. Simultaneous minimally invasive repair and lobectomy were performed successfully. The pathological diagnosis of CCLL included 5 cases of congenital cystic adenomatoid malformation (cases 2, 3, 4, 5 and 7) and 3 cases of lobar emphysema (cases 1, 6 and 8) (Table 2). The mean duration of surgery was 170 [90-360] minutes, the duration of ventilator weaning was 900 [120-2,520] minutes, and perioperative bleeding was 14.15 [6-25.93] $\mathrm{mL} / \mathrm{kg}$. There were no significant differences in the mean blood loss, the mean weaning time from mechanical ventilation and the mean hospital stay $(\mathrm{P}>0.05)$. The mean operative time (170 minutes) was extended, as expected $(\mathrm{P}=0.02)$ (Table 3). Re-examinations were conducted, and satisfaction surveys were filled out at 1, 3, 6 months and 1 year after surgery (Figure 3). With a mean follow-up of 22 months, the overall cosmetic results were good. There was one case of a surgical complication: case 5 had chest and abdominal subcutaneous emphysema that resolved on its own after 7 days. Cases 3 and 4 who had underwent simultaneous surgery for 3 years had their bars removed. No recurrence was found in both cases.

\section{Discussion}

The condition of severe PE concomitant with CCLL is not

Table 2 Patient operative data

\begin{tabular}{llllll}
\hline \multicolumn{2}{l}{ Patient No. Lung lobe involved } & Duration of surgery $(\mathrm{min})$ & Duration of ventilator weaning $(\mathrm{min})$ & Perioperative bleeding $(\mathrm{mL} / \mathrm{kg})$ & Histology \\
\hline 1 & RUL & 90 & 1,140 & 6 & 16.67 \\
2 & LLL & 160 & 360 & 10 & CLE \\
3 & LLL & 180 & 180 & 11.11 & CCAM \\
4 & LUL & 300 & 480 & 25.93 & 13.64 \\
5 & RUL & 150 & 300 & 22.22 & CCAM \\
6 & RUL & 195 & 120 & 7.69 \\
7 & RUL & 195 & 2,520 & $14.15 \pm 7.02$ \\
\hline
\end{tabular}

RUL, right upper lobe; LUL, left upper lobe; ML, right middle lobe; RUL, right upper lobe; CLE, congenital lobar emphysema; CCAM, congenital cystic adenomatoid malformation. 
Table 3 Comparison of operative data between study and control groups

\begin{tabular}{llll}
\hline Operative data & Study group [8] & Control group [30] & $P$ value $(t$-test) \\
\hline Total surgical time $(\mathrm{min})$ & $170 \pm 67.1$ & $99.67 \pm 53.9$ & 0.02 \\
Perioperative bleeding (mL/kg) & $14.15 \pm 7.02$ & $19.48 \pm 45.8$ & 0.94 \\
Duration of ventilation (mins) & $900 \pm 931.73$ & $762.67 \pm 1,017.26$ & 0.72 \\
Length of stay (days) & $16.9 \pm 5.6$ & $13.1 \pm 3.49$ & 0.11 \\
\hline
\end{tabular}

$\mathrm{P}<0.05$ indicates statistical significance.
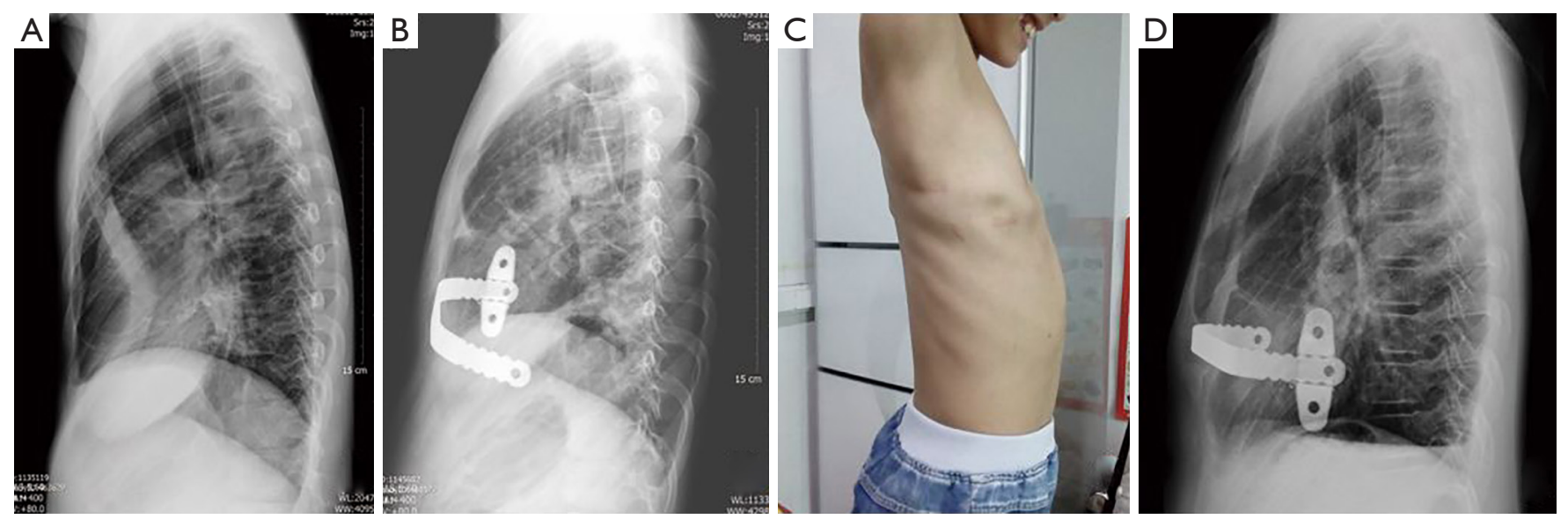

Figure 3 Pre- (A) and post-operative (B) lateral X-ray images of case 5 showing that PE was corrected. Post-operative image (C) and lateral $\mathrm{X}$-ray image (D) during follow-up (6 and 24 months, respectively) showing good cosmetic results for PE.

common. Considering the surgical trauma and anesthetic risk, the surgical treatments have been traditionally applied by stages. Compared with simultaneous surgery for the two deformities mentioned above, staged surgery sounds like shorter operation time, less perioperative blood loss, and decreased incidence of complications.

Generally, the surgical age of CCLL tends to be younger than that of PE. After lobectomy in the first stage of surgery, lung volume restriction, small-airway obstruction, and total respiratory resistance persist due to PE deformity. In other words, staged surgery could not correct the pathophysiological mechanisms of concurrent deformities completely $(7,8)$. Moreover, two separate surgeries lead to twice stress and risk for sick children (4). Last but not the least, the second-stage surgery for PE correction carries a high surgical risk due to factors such as post-operative tissue adhesions.

The simultaneous surgery appears to be an ideal solution for a complicated problem, but the most important premise is safety and efficiency. In this group, all patients had the diagnosis confirmed by CT and underwent successful lobectomy and MIRPE. Except for one patient who developed subcutaneous emphysema that resolved without treatment, there were no complications in our case series. There was no significant difference in mean blood loss, mean weaning mechanical ventilation time or mean hospital stay between this group and another group of patients who underwent lobectomy alone for CCLL in the same period. Under the premise of ensuring safety, the repair of sternal depression and cystic lung lesions deformities completely could correct the pathophysiological mechanism of this complicated situation efficiently. In consequence, simultaneous surgery might shorten the recovery phase and be considered as one kind of fast-track surgery protocol for concurrent PE concomitant with CCLL.

Although only a few cases have been reported due to the lower morbidity, comparable conclusions have been reported in recent papers concerning simultaneous correction of PE and CCLL $(9,10)$. In 2007, Metzelder et al. reported a 5-year period of MIRPE with concomitant 
thoracic interventions, including one case that underwent lobectomy for bronchopulmonary dysplasia (9). In 2015, Bilgi et al. reported two patients who underwent simultaneous MIRPE and lobectomy (10). Along with our study, no severe complications were reported in all eleven cases and excellent long-term results were achieved, including 2 patients who had the bars removed. Similarly, simultaneous surgery for $\mathrm{PE}$ concomitant with heart disease also achieved satisfactory results $(11,12)$.

There was one striking common ground in all the studies mentioned above: the repair techniques for $\mathrm{PE}$ were MIRPE. Since 1911, a variety of surgical techniques have been developed for PE correction, such as the Ravitch procedure. Over the last 20 years, MIRPE has been promoted for its advantages, including less blood loss, shorter operative times, and ease of the surgical procedure. To date, MIRPE has become standard practice worldwide because of its high satisfaction scores for correcting PE, low negative impact on cardiopulmonary function, and wide scope of applications in both pediatric and adult patients. It completely overturns the traditional theory and impressions of PE correction. In fact, it is MIRPE that enables staged lobectomy and subsequent $\mathrm{PE}$ correction.

MIRPE was initially performed at our medical center since 2007. Nowadays, the mean operative time and blood loss in the hands of seasoned surgeons were approximately 50 minutes and 5 milliliters, respectively. With regard to blood loss, duration of ventilator weaning and length-ofstay, simultaneous surgery did not increase the anesthetic risk and surgical trauma compared to data of lobectomy alone. The duration of simultaneous surgery was extended as expected, but the extension was actually the prospective second-stage surgery. Our experience indicates that simultaneous surgery including lobectomy and subsequent MIRPE is safe and reliable, but only in the hands of seasoned surgeons and be performed on selected patients. Specifically, the Haller index and respiratory tract infection may be important factors affecting surgical outcomes. Therefore, preoperative CT examination is important and should not be omitted.

With regard to the particular technique of simultaneous surgery for PE concomitant with CCLL, lobectomy was performed before MIRPE based on three technical considerations. First, the Nuss bar and stabilizer would been exposure obstacles for the lobectomy. Second, it was important to avoid the risk of the bar shifting due to intra-operative body movement. Last but not least, lobectomy was given priority due to the higher demand for peri-operative anesthesia and airway management than was needed for MIRPE.

In addition to an increased number of included patients, a striking distinguishing feature of this study was the younger age of patients than that of patients included in other related studies. In fact, infants who have undergone MIRPE have been rarely reported. Though MIRPE can be performed on a wide range of age groups, arguments on the ideal age group have lasted for years (13). What should be emphasized is that the indication for $\mathrm{PE}$ correction in this study was not cosmetic considerations but sternal depression and cardiac displacement. Surgical indications for severe PE presenting with clinical symptoms were not restricted by age (13). In this group of pediatric patients, cases 1, 2 and 8 were infants while cases 3 and 4 were preschool children. Before surgery, all patients showed clinical symptoms of recurrent respiratory tract infection, aggravated shortness of breath, and declined physical endurance. CT scan results showed significant heart displacement and the Haller indices were 3.28, 4.22, 5.99, 7.19, 5.00 in these 5 cases. Although these included patients aged 4 months to 3 years were relatively young, the indications for surgery were present. From a historical perspective, symptoms and severity were main criteria, and age was important reference for MIRPE. In the future, comprehensive evaluation of multi-index deserves further research.

One of the opposite opinions for MIRPE on the younger children was that PE severity might relieve with age. At this point, there was another noteworthy early-stage case. This patient underwent surgical treatment for PE concomitant with CCLL by stages. When the lobectomy was first performed at 7-month-old, the Haller index was 3.48. While MIRPE was performed for PE, the Haller index had gradually increased to 5.79. Before the second-stage surgery, the compression of heart had lasted and aggravated for more than 2 years. In contrast, the heart displacement of a 4-month-old infant due to cardiac compression improved immediately after the operation (Figure 4). Moreover, the 38 months of follow-up data showed that the heart displacement was corrected effectively, and recurrent respiratory tract infections and dyspnea were alleviated.

Ongoing controversy persisted regarding the age of MIRPE, particularly in younger children. Since the severity and asymmetry of $\mathrm{PE}$ deformities might increase with age (14), good long-term outcomes of MIRPE performed at a relatively early stage could be expected. However, one lesson from case 1 was that for accelerated development in childhood, the Nuss bar was significantly shorter than the thoracic transverse diameter at 6 months after surgery 

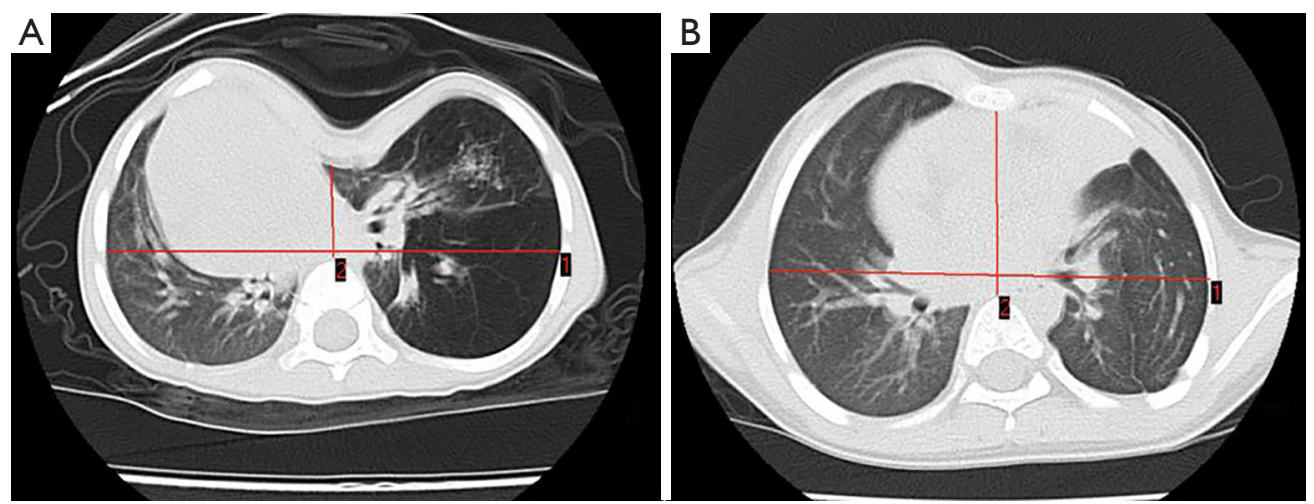

Figure 4 Pre- and post-operative CT images of case 4. The preoperative Haller index of case 4 was 4.90 (A), and it decreased to 2.42 (B) 38 months after surgery, when the bar was removed. Although the cosmetic results were not perfect, the heart displacement was corrected.
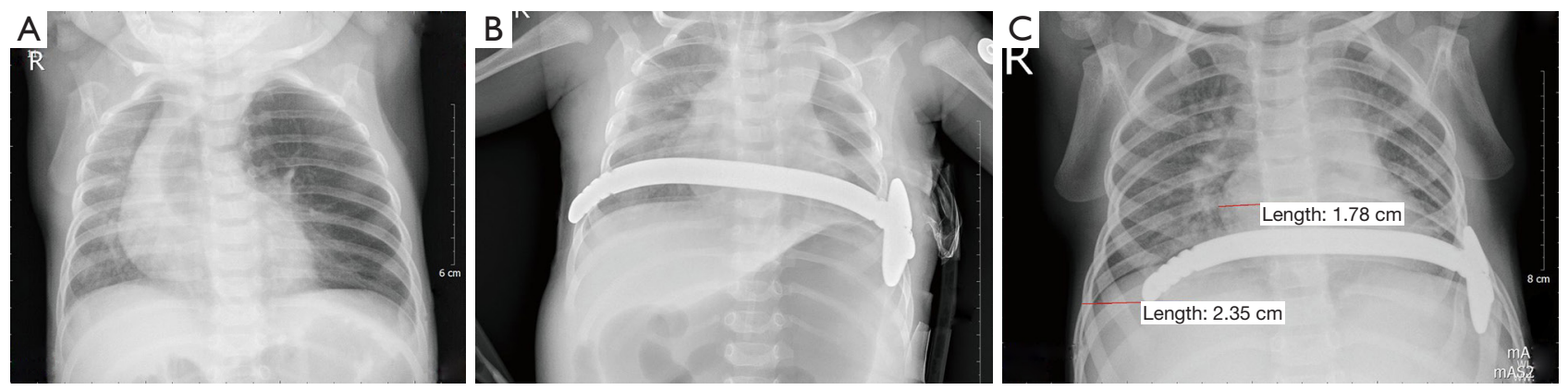

Figure 5 Anteroposterior (AP) X-ray images of case 1. (A) Preoperative image showing obvious heart displacement into the right hemithorax; (B) the heart displacement of this infant was immediately improved after surgery; (C) image at 38 months after surgery showing that the heart displacement was corrected effectively, but the bar length was $2.35 \mathrm{~cm}$ short due to the dramatic growth and development of the young child.

(Figure 5). Therefore, the surgical indications of MIRPE for younger children should be made based on rigorous evaluation.

With regard to the lesson mentioned above and the Basle experience (15), it was not the bar but the vacuum bell that was used in an 8-month-old infant with $\mathrm{PE}$ concomitant with CCLL during the perioperative period of the lobectomy. However, the time to extubation and hospital stay were 40 hours and 25 days, respectively, which were much longer than the corresponding data ( 15 hours and 16 days) reported in this study.

During follow-up, the bars in two cases were removed. The preoperative Haller indices in these two cases were 4.65 and 4.2 , and the post-operative Haller indices were 2.53 and 2.10, respectively. Although the cosmetic results of the chest wall appearance were not perfect in case 4 , the heart displacement was corrected well (Figure 5).

\section{Conclusions}

Simultaneous minimally invasive repair and lobectomy appears to be a technically safe and reliable method for the treatment of concurrent PE and CCLLs, although further studies are needed in the long-term follow-up. From a historical perspective, symptoms and severity were main criteria, and age was important reference for MIRPE. In the future, comprehensive evaluation of multi-index deserves further research.

\section{Acknowledgements}

Funding: This work was supported by the by the State Clinical Key Specialty Construction Project (Pediatric Surgery) 2013. Grant Number:GJLCZD1301 and Guangzhou Institute of Pediatrics/Guangzhou Women and 
Children's Medical Center/ (NO: IP-2018-012).

\section{Footnote}

Conflicts of Interest: The authors have no conflicts of interest to declare.

Ethical Statement: The study was approved by the institutional review board of the medical center (No. 2016111876). Written informed consent was obtained from the patient for publication of this manuscript and any accompanying images.

\section{References}

1. Koumbourlis AC. Pectus excavatum: pathophysiology and clinical characteristics. Paediatr Respir Rev 2009;10:3-6.

2. Koumbourlis AC, Stolar CJ. Lung growth and function in children and adolescents with idiopathic pectus excavatum. Pediatr Pulmonol 2004;38:339-43.

3. Kelly RE Jr, Shamberger RC, Mellins RB, et al. Prospective multicenter study of surgical correction of pectus excavatum: design, perioperative complications, pain, and baseline pulmonary function facilitated by internet-based data collection. J Am Coll Surg 2007;205:205-16.

4. Huddleston CB. Pectus excavatum. Semin Thorac Cardiovasc Surg 2004;16:225-32.

5. Shu Q, Shi Z, Xu WZ, et al. Experience in minimally invasive Nuss operation for 406 children with pectus excavatum. World J Pediatr 2011;7:257-61.

6. Wang H, Wang FH, Liang JH, et al. Simultaneous surgery procedure of pectus excavatum and congenital

Cite this article as: Wang $\mathrm{H}$, Wang $\mathrm{FH}$, Liang JH, Liu W, Yang JZ, Guo XH, Si WY, Xia HM. Pectus excavatum and congenital cystic lung lesion: simultaneous surgery advocated. J Thorac Dis 2018;10(11):6230-6237. doi: $10.21037 /$ jtd.2018.10.8 cystic lung lesion. Asvide 2018;5:865. Available online: http://www.asvide.com/article/view/28462

7. Miller DR, Pugh DM. Repair of ascending aortic aneurysm and aortic regurgitation complicated by acute cardiac compression by pectus excavatum in Marfan's syndrome. J Thorac Cardiovasc Surg 1970;59:673-84.

8. Shamberger RC, Welch KJ, Castaneda AR, et al. Anterior chest wall deformities and congenital heart disease. J Thorac Cardiovasc Surg 1988;96:427-32.

9. Metzelder ML, Ure BM, Leonhardt J, et al. Impact of concomitant thoracic interventions on feasibility of Nuss procedure. J Pediatr Surg 2007;42:1853-9.

10. Bilgi Z, Ermerak NO, Bostanc1 K, et al. Feasibility and complications in concomitant lung resection with minimally invasive repair of pectus excavatum. Ann Thorac Surg 2015;100:707-9.

11. Sacco Casamassima MG, Wong LL, Papandria D, et al. Modified Nuss procedure in concurrent repair of pectus excavatum and open heart surgery. Ann Thorac Surg. 2013;95:1043-9.

12. Schmidt J, Redwan B, Koesek V, et al. Pectus excavatum and cardiac surgery: simultaneous correction advocated. Thorac Cardiovasc Surg 2014;62:238-44.

13. Kelly RE, Goretsky MJ, Obermeyer R, et al. Twentyone years of experience with minimally invasive repair of pectus excavatum by the Nuss procedure in 1215 patients. Ann Surg 2010;252:1072-81.

14. Chang PY, Zeng Q, Wong KS, et al. A Cross-Sectional Study of Chest Wall Development in Patients with Pectus Excavatum. Thorac Cardiovasc Surg 2015;63:433-6.

15. Haecker FM. The vacuum bell for conservative treatment of pectus excavatum: the Basle experience. Pediatr Surg Int 2011;27:623-7. 